\title{
THRESHOLD-FREE PATTERN-BASED LOW BIT RATE VIDEO CODING
}

\author{
Manoranjan Paul and Manzur Murshed \\ Gippsland School of Information Technology, Monash University, Churchill, Vic-3842, Australia \\ E-mail: \{Manoranjan.paul, Manzur.Murshed\}@infotech.monash.edu.au
}

\begin{abstract}
Pattern-based video coding (PVC) has already established its superiority over H.264 in low bit rate areas because of an extra pattern-mode to segment out the arbitrary shape of the moving region in macroblock. To determine the pattern-mode, the PVC however uses three thresholds which sometimes depend on the content of the video sequences. Thus, the best performance of the PVC may not be achieved using the pattern-mode. In this paper, a novel PVC scheme is proposed which does not use any thresholds to determine the pattern-mode and hence more efficient performance is achieved without knowing the content of the video sequences. In addition, efficient pattern similarity metric and new Lagrangian multipliers are also developed. The experimental results confirm that this new scheme significantly improves the quality compared to the existing PVC as well as H.264 video coding standard.
\end{abstract}

\section{INTRODUCTION}

H.264/AVC [1] has introduced variable block size from $16 \times 16$ to $4 \times 4$ pixels motion estimation (ME) and motion compensation (MC) coding idea to approximate the shape of the moving objects within the macroblock (MB) more accurately compared to the previous video coding standards. This requires a separate motion vector for each partition, with the choice of partition size and the number of partition types. It can be easily observed that the possibility of choosing smaller partition sizes diminishes as the target bit rate is lowered. Consequently, coding efficiency improvement due to $\mathrm{MB}$ partitioning can no longer be realized for a low bit rate target for the application of video conference, video telephony where larger partition sizes have to be chosen in most cases to keep the bit-rate in check but at the expense of inferior shape approximation. Moreover, a moving object is not always approximate to the rectangular pattern which is assumed in H.264 ME and MC.
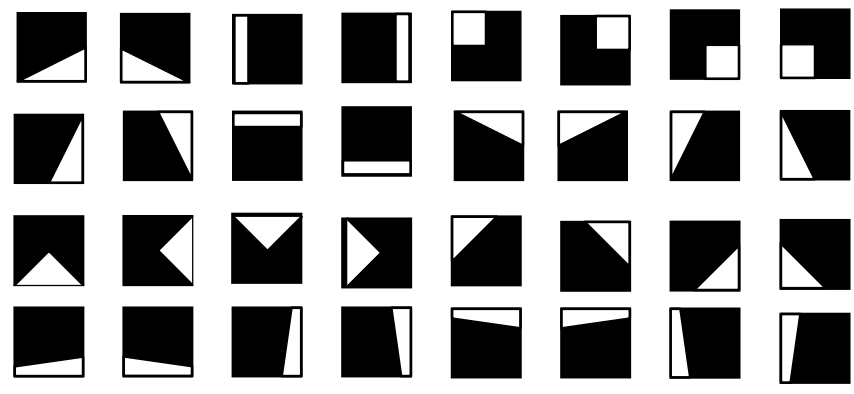

Figure 1: The pattern codebook of 32 regular shaped, 64-pixel patterns (numbering from left to right and top to bottom), defined in $16 \times 16$ blocks, where the white region represents 1 (motion) and black region represents 0 (no motion).

Wong et al. [2] recently introduced coding scheme using eight pattern templates by exploiting the principle of the MPEG-4 standard in partitioning the MBs via a simplified segmentation process that again avoided handling the exact shape of the moving objects, so that popular MB-based ME \& MC techniques could be applied. Paul et al. [3][4][5] extended and embedded this approach into the H.264 standard as an extra mode. This pattern-based video coding (PVC) algorithm focused on the moving regions (a binary representation of pixels in motion) of the MBs, through the use of a set of regular 64-pixel pattern templates (see Figure 1), from a pattern codebook (PC). If in using similarity metric, the moving region (MR) of an MB is well covered by a particular pattern, and then the $\mathrm{MB}$ can be motion estimated and compensated by considering only the 64 pixels of that pattern, with the remaining 192 pixels being skipped as static background. The MBs are also motion estimated and compensated by the other modes and then final mode is selected using an optimization cost function popularly known as the Lagrangian multiplier (LM). It is defined as active-region MB (RMB). A RMB can therefore, theoretically, be coded by around one-fourth bit rates and sometimes slightly higher distortion due to the poor pattern approximation of the MR compared to the other modes. Since the Lagrangian optimization cost function is biased to the bit rate as low bit rate range, a significant number of MBs are coded using the pattern-mode which improves the rate-distortion performance compared to the H.264 standalone standard. Note that this number gradually decreases with the increasing of bit rates. A pattern approximation example is given in Figure 2.

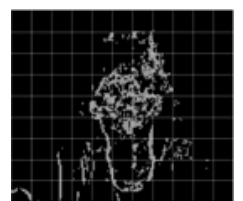

(a)

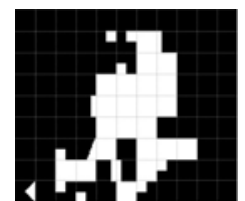

(b)
Figure 2: Pattern approximation for the Miss America, (a) detected moving region between frame one and two, and (b) pattern approximation by the PVC algorithm using 32 patterns.

The existing PVC scheme uses three parameters (or thresholds) to process the pattern-mode for $\mathrm{MB}$ in the i) generation of binary MR, ii) identification of MB for pattern-mode based on the MR size, and iii) determination of pattern similarity with the MR. The first threshold (pixel intensity difference threshold) is used to generate the binary MR of a MB from the current and reference frame. A pixel position is declared moving point, if the co-located pixel intensity difference between current and reference MB is more than a threshold. The collection of moving points in a MB is defined as MR of that MB. The problem with this MR definition is that it does not differentiate low to high intensity changes. When the bestmatched similar pattern does not cover the whole MR of a MB, there is a possibility to exclude some high moving points instead of low moving points. Thus, by treating all moving points equally degrades the performance. The second threshold (moving region size threshold) is used to eliminate MBs unsuitable for pattern representation at an early stage. Third threshold (pattern similarity threshold) is used to avoid dissimilar MR with the best-matched pattern being processed in pattern-mode which is not ultimately classified as RMB using optimization function, though the size of MR is within the range. Although these thresholds were set after performing sensitivity analysis on a large number of standard and non-standard video sequences, they impinge in extracting the best achievable rate-distortion performance as unsuitability/suitability measure of MBs have no direct bearing on the ultimate ratedistortion performance. 
In this paper, a novel PVC scheme is proposed which does not require any parameters/thresholds to determine the pattern-mode and the ultimate mode selection is determined without knowing the content of the video sequences. We eliminated the first threshold using a new similarity measure. Note that, as the best pattern selection process relies on solely the similarity/proximity measures, it is not guaranteed that the best pattern will always result in maximum compression, which also depends on residual errors and the length of pattern identification numbers. This paper exploited this possibility by introducing additional pattern modes that select the pattern in order of similarity/proximity ranking. This work has led us in enhancing the generic pattern based coding technique to its ultimate state as explained later. The last two thresholds are eliminated by allowing all MBs to be considered while using multiple pattern modes in H.264 embedding. Computational complexity is checked by using already known motion vector in H.264 for pattern modes. In addition, a new LM is also developed. The experimental results confirm that this new scheme successfully improves the rate-distortion performance compared to the existing PVC as well as the H.264 standard without increasing the computational complexity compared to the existing real time PVC approach.

\section{EXISTING PVC}

For the seek of completeness, this section briefly describe the existing pattern-based video coding. The pre-defined thirty two patterns approximate the $\mathrm{MR}$ in an $\mathrm{MB}$ with white regions representing the $\mathrm{MR}$ and black regions representing the static background. If $C_{k}(x, y)$ and $R_{k}(x, y)$ denote the $k^{\text {th }} \mathrm{MB}$ of the current and reference frames of a video sequence, each of size $W$ pixels $\times H$ lines, respectively of a video sequence, where $0 \leq x, y \leq 15$ and $0 \leq k<W / 16 \times H / 16$, the MR $M_{k}(x, y)$ of the $k^{\text {th }}$ $\mathrm{MB}$ in the current frame is obtained [2] as follows:

$$
M_{k}(x, y)= \begin{cases}1 & \text { if }\left|C_{k}(x, y) \bullet B-R_{k}(x, y) \bullet B\right|>T_{V} \\ 0 & \text { otherwise }\end{cases}
$$

where $B$ is a $3 \times 3$ unit matrix for the morphological closing operation - [7], which is applied to reduce noise, and the MR threshold $T_{v}$ is 2 [2]. As ' 1 ' indicates the MR and ' 0 ' the static region of that MB.

Let $|Q|_{\ell}$ be the total number of $\ell$ 's in the matrix $Q$.

Dissimilarity of a pattern $P_{n} \in P C$ with the MR in the $k$ th MB can be defined [4]as

$$
D_{k, n}=\left|M_{k}\right|_{1}-\left|M_{k} \wedge P_{n}\right|_{1} .
$$

Clearly, higher the similarity lower will be the value of $D_{k, n}$.

Obviously all MBs are not examined using pattern. The eligible MBs are called Candidate RMB (CRMB) and defined as $8 \leq\left|M_{k}\right|_{1}<T_{C R M B}$ and $8 \leq\left|M_{k}\right|_{1}<T_{C R M B}$, where $T_{C R M B}$ are the predefined CRMB threshold. The CRMB is classified as an RMB and its moving region is represented by a pattern $P_{i}$ such that

$$
\left.P_{i}=\arg \min _{\forall P_{n} \in \mathrm{PC}}\left(D_{k, n}\right) \mid D_{k, n}<T_{S}\right)
$$

otherwise, the CRMB is classified as normal MB which is not coded through pattern-mode. To avoid more than four $4 \times 4$ blocks of DCT calculations for 64 residual error values per RMB, these values are rearranged into an $8 \times 8$ block. It avoids unnecessary DCT block transmission. A similar inverse procedure is performed during the decoding.

It is clear that due to the above mentioned three thresholds the expected performance cannot be achieved through pattern-mode where some potential RMBs are not classified as RMBs.

\section{PROPOSED ALGORITHM}

Obviously, the purpose of the above mentioned thresholds is to reduce the number of MBs coded using pattern-mode to control the computational complexity. by coding all MBs using the patternmode eventually improves the performance but demands other issues to be solved, such as i) developing a new similarity metric which does not require binary $\mathrm{MR}$, ii) investigating the validity of the existing LM in this case, and more importantly iii) controlling the computational complexity in real time. As alluded in the Introduction section, the best pattern selection process relies on the similarity/proximity measures does not guarantee that the best pattern will always result in maximum compression because of residual errors and pattern identification numbers. To eliminate this possibility we also propose multiple pattern modes that select the pattern in order of similarity/proximity ranking.

\subsection{New Similarity Metric}

The main purpose of similarity metric to find the best-match pattern for a given MR. The existing similarity metric selects the best pattern based on the minimum non-overlapping region of binary MR between the MR and the pattern (see equation 1 and 2). When the best-matched similar pattern does not cover the whole MR of a MB, there is a possibility to exclude some high moving points instead of low moving points. Thus, by treating all moving points equally may not provide the best pattern. The proposed similarity metric does not require any pixel intensity difference threshold as it does not need any binary MR for selecting the best pattern. It determines the best pattern based on the absolute difference between current and colocated reference MBs. If $C_{k}(x, y)$ and $R_{k}(x, y)$ denote the $k^{\text {th }} \mathrm{MB}$ of the current and reference frames of a video sequence. The absolute error matrix is calculated as follows:

$$
E_{k}(x, y)=\left|C_{k}(x, y)-R_{k}(x, y)\right| .
$$

The best pattern for a MR is selected as follows:

$$
P_{i}=\arg \max _{\forall P_{n} \in \mathrm{PC}}\left(S_{k, n}\right)
$$

where $S_{k, n}(x, y)=\sum_{x=0}^{15} \sum_{y=0}^{15}$
\begin{tabular}{|c|c|}
\hline 0 & 3 \\
\hline 2 & 8 \\
\hline
\end{tabular}

(a)

\begin{tabular}{|l|l|}
\hline 0 & 0 \\
\hline 1 & 0 \\
\hline \multicolumn{2}{|c|}{ (c) }
\end{tabular}

\begin{tabular}{|l|l|}
\hline 0 & 0 \\
\hline 0 & 1 \\
\hline \multicolumn{2}{|c|}{ (d) }
\end{tabular}

Figure 3: Pictorial description of new similarity metric using 2 $\times 2$ pixel block; (a) error matrix, (b) binary matrix, (c)-(f) four patterns.

The other advantage of this similarity metric over existing one is that it considers the most dominating moving pixels where pixel intensity difference is higher. Obviously the same pattern will be selected in both cases where MR of a MB is fully covered by the best pattern otherwise different patterns may be selected. An example is given in Figure 3 where an error matrix and a binary matrix are formed using (4) and (1) respectively. Pattern in Figure 3(d) or (e) will be selected using the previous similarity metric, whereas pattern in Figure 3(d) will be selected by the new similarity metric. Obviously pattern in Figure 3(d) is the best choice as it captures the most dominating moving pixel.

\subsection{Multiple Pattern Modes}

As alluded to in the Introduction section, the best pattern selection process relies on the similarity/proximity measures does not guarantee that the best pattern will always result in maximum compression because of residual errors and pattern identification 
numbers. To eliminate this possibility we also propose multiple pattern modes that select the pattern in order of similarity/proximity ranking. Since the similarity/proximity measure is a good estimator, we only consider higher ranked patterns. Obviously it will increase the computational complexity. Computational complexity is checked by using already known motion vector determined by $16 \times 16$ mode. From the experimental results we observed that when the MR is not fully covered by the pattern, the pattern mode using this motion vector provides better result. Thus, in average case we may not sacrifice the quality significantly. Moreover, allowing multiple pattern modes we overcome this problem.

The Figure 4 shows the percentages of RMBs finally selected by the k-th best similar patterns using two different similarity matrices. From the figure it is clear that in both cases the best similar pattern captures most of the RMBs. It also clear that proposed similarity metric capture performs better as the best pattern captures $75 \%$ RMBs while the counter part captures 54\%.

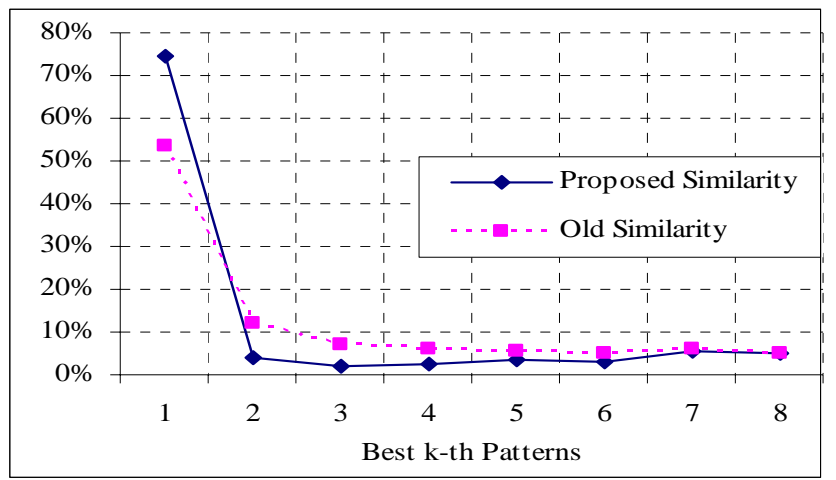

Figure 4: Percentages of RMBs finally selected by the k-th best similar patterns using proposed and old similarity matrices for Miss America video sequence where $\mathrm{QP}=30$.

\subsection{New Lagrangian Multiplier}

The Lagrangian multiplier is used to select the final mode among all modes. The Lagrangian multiplier is first determined based on the experimental results in [8] and defined as $\lambda=0.85 \times 2^{(Q P-12) / 3}$ where $Q P$ stands for quantization parameter. During the motion estimation and encoding processes, all modes of every MB are examined and the resulting rates, $R\left(m_{i}\right)$, and the distortions, $D\left(m_{i}\right)$, are determined, where $m_{i}$ is the $i$-th mode. The Lagrangian cost function is defined as:

$$
J^{L M}\left(m_{i}\right)=D\left(m_{i}\right)+\lambda \times R\left(m_{i}\right) .
$$

where $R\left(m_{i}\right)$ is the sum of the bits for mode $m_{i}$ including the mode information, the motion vectors and the transformation coefficients, while $D\left(m_{i}\right)$ is measured as the sum of square difference (SSD) between original $\mathrm{MB}$ and corresponding reconstructed $\mathrm{MB}$ for mode $m_{i}$.

We do not expect the same LM after inclusion of multiple pattern modes as the LM is already changed from H.263 to H.264. We use the same procedure described in [8] to determine the LM. From the experimental results we conform that after including multiple pattern modes the new LM is redefined as follows:

$$
\lambda=0.4 \times 2^{(Q P-12) / 3} \text {. }
$$

We have already mentioned that a pattern mode provides less bits and sometimes higher distortion compared to the other modes. Due to this, it is biased to the bit rate. To keep the fairness to the other modes, it is rational to reduce the value of LM. The experimental results of LM determination and rate-distortion performance also justify the new value. Due to the page limit we could not provide here detail results.

\subsection{Computational Complexity}

Let $\alpha, d$, and $m$ are the MBs, total motion search points, and the number of modes for motion estimation respectively. For motion estimation the H.264 requires $m d \alpha$ operations. After motion estimation we need to calculate the operation for bits stream generation. But this operation depends on the combination of DCT coefficient and variable length code tables. To avoid this ambiguity we like to assume the operations. The researchers already claimed that motion estimation, irrespective of a scene's complexity, typically comprises more than $60 \%$ of the processing overhead required to encode an inter picture with a software codec using the DCT [9], when full search is used. From this fact we conclude that the H.264 requires $1.66 m d \alpha$ operations for encoding.

The existing PVC algorithm termed as PVCold needs to process one extra pattern mode. It requires only one-fourth motion estimation operations as the pattern size is one-fourth of MB. Thus, the PVCold algorithm requires $1.66 m d \alpha+0.13 d \alpha$ operations by assuming 30\% MBs are examined by pattern mode. On the other hand, the proposed method does not require any motion estimation operations as it uses already available motion vector. It only requires operations for encoding the MB using pattern which is one-fourth of MB. Thus the proposed PVC termed as PVCnew algorithm requires $1.66 m d \alpha+0.15 \alpha \delta$ operations by assuming $\delta$ number of pattern modes will be processed. The PVCold algorithm requires $0.13 d \alpha$ more operations and the PVCnew algorithm requires $0.15 \alpha \delta$ more operations compared to the H.264 standard. Thus, the PVCold and the PVCnew algorithms require $1 \%$ and $0.18 \%$ more operations compared to the H.264 standard using full search and eight pattern modes. Moreover, due to the pattern mode some MBs are not processed by subblock modes (such as $8 \times 4,4 \times 8$, and $4 \times 4$ ), as a result the PVCnew require less operations in those cases. Thus, we can conclude that our new approach requires almost same operations compared to the H.264.

\section{SIMULATION RESULTS}

The PVCold, PVCnew, and H.264 algorithms are implemented based on the Baseline profile of H.264/AVC [1] with full search motion estimation, up to fraction-Pel accuracy, and group of picture size is 12 for number of standard QCIF [4] video sequences with 15 frames per second (defined as $\mathrm{Hz}$ ). The experimental results show that new threshold free scheme with eight pattern modes outperforms the existing PVC algorithm and the H.264 (see Figure 5) by at least $0.5 \mathrm{~dB}$ peak signal to noise ratio (PSNR) [6] at 64 kilo bits per second (kbps) for all video sequences which is significant improvement.

\section{CONCLUSIONS}

In this paper a new pattern-based video doing algorithm is proposed which successfully avoided three thresholds that have been used in previous algorithm. By avoiding the thresholds, this algorithm fully exploited the well known Lagrangian optimization function to select the best mode. In this paper a new similarity metric, multiple pattern modes, and a modified Lagrangian multiplier are also proposed. This new PVC algorithm with efficient similarity metric, multiple pattern modes, and modified Lagrangian multiplier has improved the image quality by $0.5 \mathrm{~dB}$ and $1.0 \mathrm{~dB}$ compared to the existing pattern-based algorithm and the H.264 standard in low bit rates respectively.

\section{REFERENCES}

[1] ITU-T Rec. H.264/ISO/IEC 14496-10 AVC. Joint Video Team (JVT) of ISO MPEG and ITU-T VCEG, JVT-G050, 2003. 
[2] Wong, K.-W., K. -M. Lam, and W. -C. Siu, "An efficient low bit-rate video-coding algorithm focusing on moving regions," IEEE Transaction on Circuits and Systems for Video Technology, 11(10), 1128-1134, 2001.

[3] Paul, M., M. Murshed, and L. Dooley, “A real-time pattern selection algorithm for very low bit-rate video coding using relevance and similarity metrics," IEEE Transaction on Circuits and System for Video Technology, 15(6), 753-761, June, 2005.

[4] Paul, M., M. Murshed, and L. Dooley, "A new efficient similarity metric and generic computation strategy for pattern-based very low bit-rate video coding," Proc. of IEEE Int. Conference of Acoustics, Speech, and Signal Processing vol. 3, pp. 165-168, 2004.

[5] Paul, M. and M. Murshed, "Efficient H.264/AVC video encoder where Pattern is Used as Extra Mode for Wide Range of
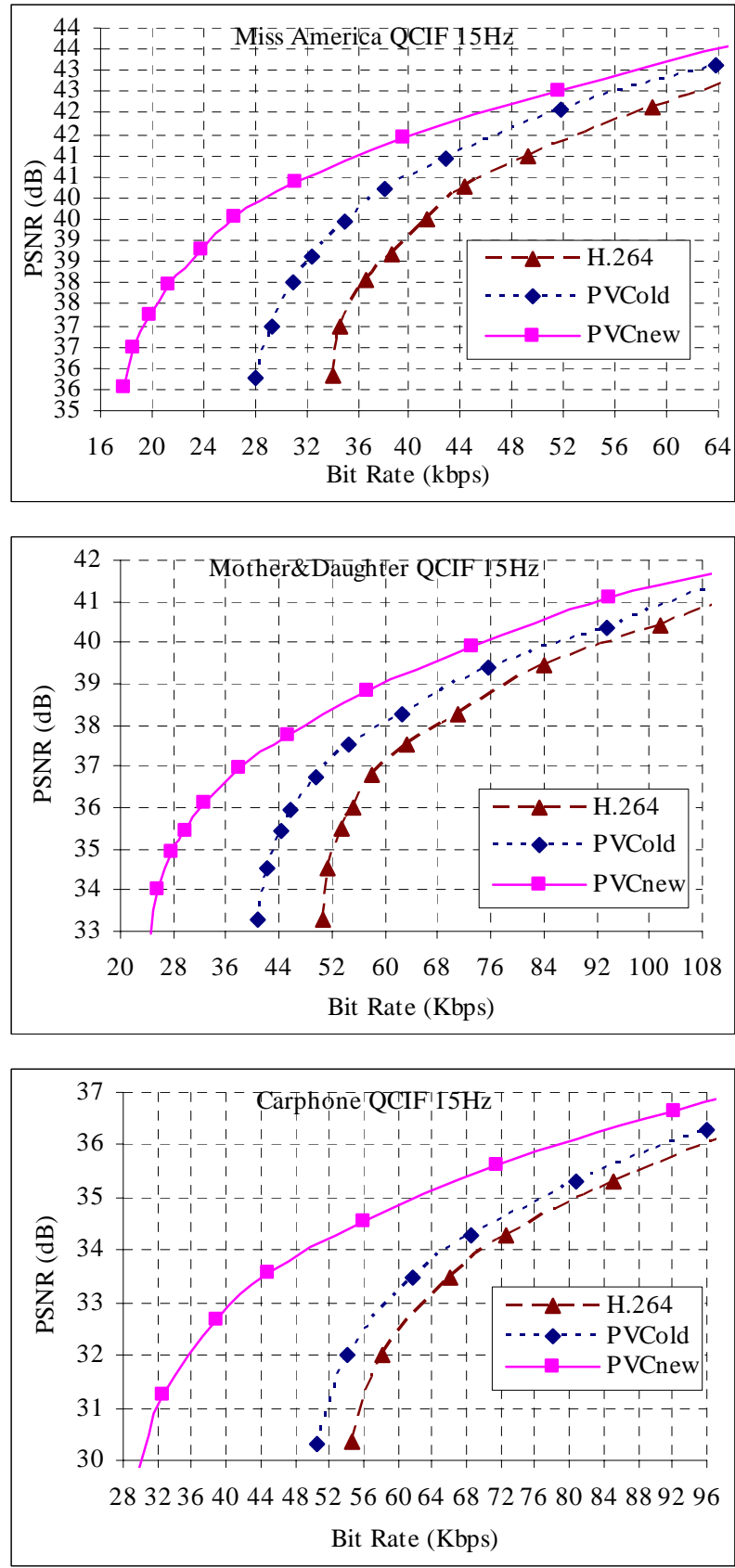

Figure 5: Rate-Distortion curves for six standard video sequences.
Video Coding,” LNC, Springer-Verlag, vol. 4352, pp. 353-362, 2007.

[6] Richardson, I. E. G., "H.264 and MPEG-4 video compression," Wiley Press, 2003.

[7] Maragos, P., "Tutorial on advances in morphological image processing and analysis,” Opt. Eng., 26(7), 623-632, 1987.

[8] Weigrand, T., H. Schwarz, A. Joch, and F. Kossentini, "Ratecontrained coder control and comparison of video coding standards," IEEE Transaction on Circuits and Systems for Video Technology, 13 (7), 688-702, 2003.

[9] Shanableh, T. and M. Ghanbari, "Heterogeneous video transcoding to lower spatio-temporal resolutions and different encoding formats,” IEEE trans. on multimedia, 2(2), 101-110, 2000.
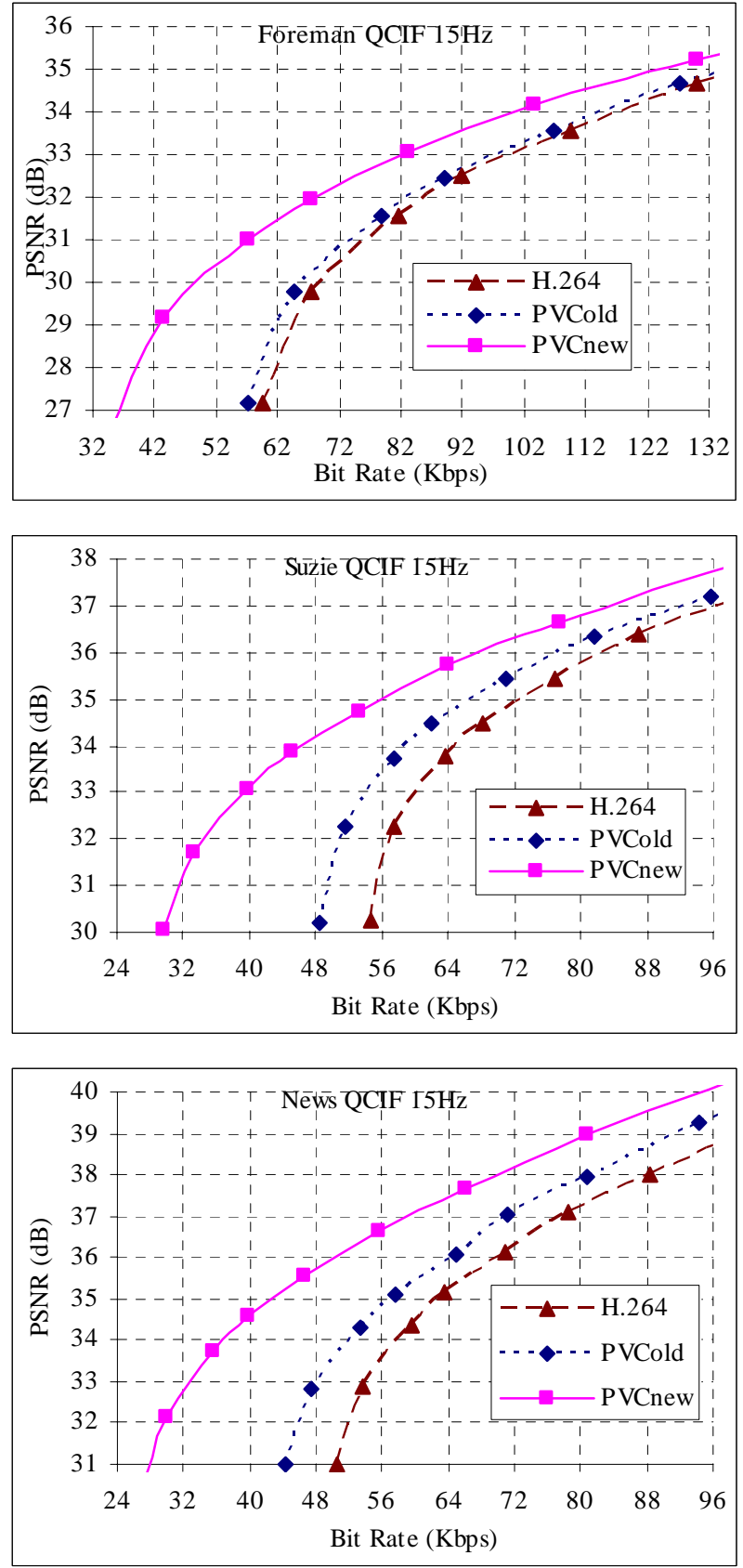